Herz 2012 · 37:815-816

DOI 10.1007/s00059-012-3721-y

Published online: 21. November 2012

(c) Urban \& Vogel 2012

B. Maisch

"HERZ/Cardiovascular Diseases", Marburg

\title{
Nothing new in heart failure? A current misconception!
}

\section{Animal models}

ure, after the abundance of imaging modalities to assess left ventricular (LV) function, e.g., color flow-Doppler, cardiac magnetic resonance imaging (MRI), computed tomography (CT), and positron emission tomography (PET), after established biomarkers to assess heart failure (BNP and others), necrosis (troponins), inflammation (CRP, cytokines), and fibrosis (Gal-3, PIP) is there anything new as we move from 2012 to 2013 in the etiology, diagnosis, and treatment of heart failure and dilated cardiomyopathy(DCM) [1]?

In issue 6 of HERZ this year, we reexamined some of the most recent developments in experimental genetic models of heart failure, from zebrafish and mice by Kloos, Katus, and Meder [2] and patients with dilated cardiomyopathy by Ruppert and Maisch [3] and Wicks and Elliot [4], to the diagnosis and treatment of giant cell myocarditis by Cooper and ElAmm [5], viral myocarditis by Kühl et al. [6], and autoreactive myocarditis by Maisch and Pankuweit [7], of right ventricular cardiomyopathy by Thiene et al. [8], restrictive cardiomyopathy by Zwas et al. [9], as well as the remodeling and adverse remodeling process after myocardial infarction by Brenner and Ertl [10]. Biomarkers of inflammation, particularly interleukin 10, were examined by Izumi and Nishii [11]. We called it"a never-ending story" [12], which is continued in this issue of HERZ. The current issue selects again hotspots of the pathogenetic process and of treatment options in heart failure in general and in inflammatory dilated cardiomyopathy in particular.
In inbred murine models of myocarditis, Matsumori reports on the natural history, on the pathogenesis, as well as on diagnostic methods and therapeutic or preventive interventions. In murine encephalomyocarditis (EMC) virus-induced disease, full-blown inflammation can be observed in the heart of the animals. However, although much can be learned about murine cardiac inflammation, unfortunately the EMC virus does not affect humans. Furthermore, autoimmune myocarditis was induced in mice lacking the T-cell receptor costimulatory molecule PD-1. They spontaneously develop troponin-liberating necrotizing autoimmune myocarditis along with the production of high titers of antibodies against cardiac Troponin I (cTnI) itself. When these antibodies were transferred to healthy mice they in turn induced myocardial dysfunction. A novel finding was that serum levels of free immunoglobulin light chains (FLC) were increased in murine EMC myocarditis, and exogenously given FLC inhibited viral replication and improved the histological myocarditis phenotype. This would fit nicely to the benefit of intravenous infusion of immunoglobulins in the treatment of viral myocarditis in humans [1].

\section{Genetics}

Serio et al. emphasize that familial dilated cardiomyopathy (F-DCM) describes a clinically and genetically heterogeneous group of diseases, which are mostly of autosomal-dominant inheritance but have a similar phenotype to left ventricular dilatation and dysfunction.

\section{Inflammation and immunoreactivity}

Noutsias et al. describe in human myocardial inflammation the differential expression of Th1, Treg, and CTL-related markers, while no major role could be found for Th17 cells. They also found a T-cell receptor Vbeta dominance, suggesting an antiviral specificity of virusinduced T-cell responses.

Staab et al. review their own data and those of others, which show that a single nucleotide polymorphism (SNP) in the gene encoding the cytotoxic T-lymphocyte antigen 4 (CTLA4) is associated with the diagnosis of DCM. CTL4 is an inhibitory receptor molecule expressed on activated $\mathrm{T}$ lymphocytes, where it competes with the costimulatory $\mathrm{CD} 28$ receptor in the binding to $\mathrm{B} 7$ receptors on the surface of antigen-presenting cells. The association between CTLA4 genotypes and DCM suggests that genetic factors contribute to both unbalanced immune responses in the myocardium and the development of LV dysfunction

Among the more than 50 anticardiac antibodies described so far in cardiac inflammation and dilatation, autoantibodies to the membrane of cardiocytes [12] and autoantibodies that target $G$ proteincoupled cardiac membrane receptors, such as the beta-receptor, are thought to play a key role in the development of heart failure. Their incidence and function in vitro and in vivo are reviewed by Boivin-Jahns et al. in this issue. Remarkably, clinical pilot studies suggest that 
they might negatively affect survival in heart failure patients.

Baandrup reports on eosinophilic myocarditis also known as endomyocarditis fibroplastica Löffler, which is rare disease with a well-described pathway of inflammation, thrombus formation, and fibrosis caused by the activation of eosinophilic granulocytes and the release of eosinophilic granules, especially the eosinophilic cationic proteins. In hypereosinophilic syndromes and Löffler's endomyocardial disease, the eosinophilia is still"idiopathic."

\section{Treatment of inflammatory dilated cardiomyopathy}

Whereas immunosuppression in eosinophilic, granulomatous, giant cell, or lymphocytic myocarditis in cardiac inflammation associated with connective tissue disease or with rejection after heart transplantation is fairly well established, there was no consensus on its therapeutic role for lymphocytic inflammatory cardiomyopathy [13] until recently when data from the ESETCID trial $[1,7]$ and the TIMIC studies were reported. In the TIMIC trial, which is reviewed here by Frustaci and Chimenti, the benefit of immunosuppression after 6 months of treatment was clearly documented on the surrogate markers of cardiac function and dilatation.

\section{Device therapy in heart failure and heart transplantation}

The Marburg Cardiomyopathy Study revealed that LV ejection fraction is the only significant independent arrhythmia risk predictor in DCM patient populations. Prophylactic ICD therapy became a class I indication based on the favorable results of the SCD-HeFT Trial in patients with DCM of NYHA class II or III heart failure and an LV ejection fraction of $35 \%$ or less despite optimized medical therapy. Prophylactic ICD therapy combined with cardiac resynchronization became the standard device therapy in DCM patients with complete left bundle branch block. These obvious beneficial effects as well as unresolved issues of prophylactic ICD therapy are described by Grimm.
Moosdorf provides and update on the current status of the implantation of cardiac assist devices and heart transplantation. The artificial heart is no longer merely a bridge to transplantation; in patients older than 65 years it is a life-prolonging destination therapy.

\section{Controversial issues}

Tschöpe and Lam take up the topic of heart failure with preserved ejection fraction (HFPEF), which is responsible for half of the disease burden of heart failure worldwide. Its pathophysiology has been associated with diastolic dysfunction, thus labeling it as "diastolic heart failure." Current evidence suggests, however, that heterogeneous mechanisms contribute to HFPEF. This may explain why in contrast to systolic heart failure (HFREF, heart failure with reduced ejection fraction), where the unloading of the ventricle is the driving therapeutic principle, there is no proven therapy for HFPEF.

Diabetic cardiomyopathy is a controversial entity, too, that starts with hypertrophy without hypertension and diastolic dysfunction. Seferovic et al. see hyperglycemia, AGEs (advanced glycation end products), and insulin resistance as the main contributors to this still underestimated syndrome in diabetics. Of note, in a recent overview in HERZ a broader concept with four stages was suggested, in which apart from diastolic dysfunction systolic heart failure also develops due to microvascular disease, increased influence of infection and microbial agents and, finally, cardiac ischemia and infarction[14].

Nothing new in heart failure? All contributions in this issue underline clearly that this is a misconception. There is much more to expect now and in the future. Thus, the "never-ending story of heart failure" still has an open end!

\section{Corresponding address}

Prof. Dr. B. Maisch

"HERZ/Cardiovascular Diseases"

Feldbergstr. 45, 35043 Marburg

Germany

Bermaisch@aol.com

\section{References}

1. Maisch B, Noutsias M, Ruppert V, Richter A, Pankuweit S (2012) Cardiomyopathies-classifications, diagnosis, and treatment. Heart Failure Clinics: 53-78

2. Kloos W, Katus HA, Meder B (2012) Genetic cardiomyopathies - lessons learned from men, mice and zebrafish. Herz 37:612-618

3. Ruppert V, Maisch B (2012) Molecular signatures and the study of gene expression profiles in inflammatory heart diseases. Herz 37:619-626

4. Wicks EC, Elliot PM (2012) Genetics and metabolic cardiomyopathies. Herz 37:598-611

5. Cooper LT, ElAmm C (2012) Giant cell myocarditis: diagnosis and treatment. Herz 37:632-636

6. Kühl U, Rohde M, Lassner D, Gross UM, Escher F, Schultheiss HP (2012) miRNA as activity markers in Parvo B19 associated heart disease. Herz 37:637-643

7. Maisch B, Pankuweit S (2012) Current treatment options in (peri)myocarditis and inflammatory cardiomyopathy Herz 37:644-656

8. Thiene G, Rigato I, Pilichou K, Corrado D, Basso C (2012) Arrhythmogenic right ventricular cardiomyopathy: what is needed for a cure? Herz 37:657-663

9. Zwas DR, Gotsman I, Admon D, Keren A (2012) Advances in the differentiation of constrictive pericarditis and restrictive cardiomyopathy. Herz 37:664-674

10. Brenner S, Ertl G (2012) Remodelling and adverse remodelling in CAD. Herz 37:590-597

11. Izumi T, Nishii M (2012) Diagnostic and prognostic biomarkers in acute myocarditis: interleukin-10. Herz 37:627-631

12. Maisch $B$ (2012) Heart failure-a never ending story. Herz 37:589

13. Mason JW, O'Connell JB, Herskowitz A, Rose NR, McManus BM, Billingham ME, Moon TE (1995) A clinical trial of immunosuppressive therapy for myocarditis: the Myocarditis Treatment Trial Investigators. N Engl J Med 333:269-275

14. Maisch B, Alter P, Pankuweit S (2011) Diabetic cardiomyopathy — fact or fiction? Herz-36:102-115 